\title{
Case of an Increase in Prothrombin Time-International Normalized Ratio by Interaction Between Warfarin and Baloxavir Marboxil in a Patient on Implantable Ventricular Assist Device
}

Keiko Kurosawa ${ }^{a}$, Shinya Takasaki ${ }^{a}$, Hisaki Suzuki ${ }^{a}, Y$ uji Sato ${ }^{a}$, Masatoshi Akiyama $^{\mathrm{b}}$, Miki Akiba $^{\mathrm{c}}$, Yoshikatsu Saiki $^{\mathrm{b}}$, Nariyasu Mano ${ }^{\mathrm{a}}$

aDepartment of Pharmaceutical Sciences; ${ }^{\mathrm{b}}$ Department of Cardiovascular Surgery, ${ }^{\mathrm{c}}$ Organ Transplantation Center, Tohoku University Hospital, Sendai, Miyagi Japan

Corresponding Author: Shinya Takasaki, Department of Pharmaceutical Sciences, Tohoku University Hospital, 1-1 Seiryo-machi, Aoba-ku, Sendai, Miyagi, Japan; TEL: (+81)-022-717-7540; Fax: (+81)-022-717-7545; email: shinya_takasaki@tohoku.ac.jp

Received, November 14, 2020; Revised, December 21, 2020; Accepted, January 15, 2021; Published, January 19, 2021

\begin{abstract}
Background: Baloxavir marboxil (BM) is a novel drug with a cap-dependent endonuclease inhibitory action for influenza A or B; it is highly safe and requires just a single oral dose. Patients with severe heart failure use implantable ventricular assist device (iVAD) until transplantation, but they have an increased risk of thrombosis development. Their warfarin is administered based on point-of-care testing (POCT) with a strict control of prothrombin time-international normalized ratio (PT-INR). Case report: Here, we report a case of a patient with iVAD whose PT-INR was significantly increased from the target range after BM administration. The patient was a 45-year-old man and transplanted with iVAD; warfarin treatment was started when his PT-INR target range was 3.0-3.5. At home, he frequently self-measured PT-INR by POCT and precisely controlled the warfarin dose. He had a fever, was diagnosed with influenza A and was administered BM $40 \mathrm{mg}$. Thereafter, his PT-INR continued to increase, reaching 4.8 on day 12 of BM administration, exceeding his target range; warfarin was skipped for 1 day. In this case, based on the history of BM administration and clinical course, the increase in PT-INR could be due to BM. Considering the interaction between warfarin and BM, we suspected a possibility of competition for protein-binding sites. Increased PTINR in the patient was detected early by POCT and thus severe bleeding was avoided. Conclusion: Strict monitoring of PT-INR when using BM in patients taking warfarin is of clinical importance.
\end{abstract}

\section{INTRODUCTION}

Baloxavir marboxil (BM) is a novel drug with a capdependent endonuclease inhibitory action for influenza A or B [1-3]. The clinical advantages of $\mathrm{BM}$ are it is highly safe and requires just a single oral dose [1-3]. In a recent trial, BM was shown to be effective and safe for patients at a high risk of developing influenza-related complications such as asthma and chronic lung disease, endocrine disorders, and heart diseases [3]. Therefore, in the future, BM will be more frequently used in high-risk patients.

In Japan, patients suffering severe heart failure and requiring transplants have a limited number of organ donors; moreover, the waiting time before a heart transplant is long. Therefore, an implantable ventricular assist device (iVAD) is used as a bridge to transplant [4]. Patients with iVAD require postoperative anticoagulant therapy and use warfarin . Their prothrombin time-international normalized ratio (PT-INR), an indicator of warfarin efficacy, is precisely controlled [5]. Warfarin poses a clinical problem because the PT-INR fluctuates due to the drug's interactions with other drugs and vitamin $\mathrm{K}$ intake [6-9]. Here, we report a case of a patient with iVAD whose PT-INR was significantly increased from the target range with the intake of BM.

\section{CASE REPORT}

The patient was a 45 -year-old man (height $171.9 \mathrm{~cm}$ and weight $71.2 \mathrm{~kg}$ ). He was transplanted with iVAD for severe heart failure and administered warfarin when his PT-INR target range was 3.0-3.5. At home, patients with iVAD must frequently self-measure PT-INR by point-of-care testing (POCT) and precisely control their warfarin dose, and so did our patient. He had a fever of $38^{\circ} \mathrm{C}$; he was examined by a physician. He was tested negative for influenza, was prescribed dextromethorphan hydrobromide hydrate and acetaminophen tablets. On the subsequent day, he visited the doctor again because 
his fever rose to $39.4^{\circ} \mathrm{C}$. He was diagnosed with influenza $\mathrm{A}$ and was administered a single dose of BM $40 \mathrm{mg}$. The changes in the PT-INR and warfarin dose before and after administering BM are shown in Figure 1. His PT-INR was 3.3 at 7 days before administering BM and warfarin was continued at the dose of $4 \mathrm{mg} /$ day. On the day of BM administration, his PT-INR was 2.4, which was below the target range; his warfarin dose was increased to 4.25 $\mathrm{mg} /$ day. On day 3 of BM administration, his fever was resolved, but his PT-INR was further reduced to
2.2 and therefore, his warfarin dose was increased to $5 \mathrm{mg} /$ day. On day 5 of BM administration, his PTINR reached the target range of 3.0 and his warfarin dose was reduced to $4.75 \mathrm{mg} /$ day. On day 8 of BM administration, his PT-INR was increased to 4.2 and his warfarin dose was reduced to $4 \mathrm{mg} /$ day. On day 12 of BM administration, his PT-INR significantly exceeded the target range of 4.8 and warfarin was discontinued. Warfarin was discontinued for 1 day, then warfarin was resumed at $2 \mathrm{mg} / \mathrm{day}$, and its dose was adjusted while monitoring his PT-INR.

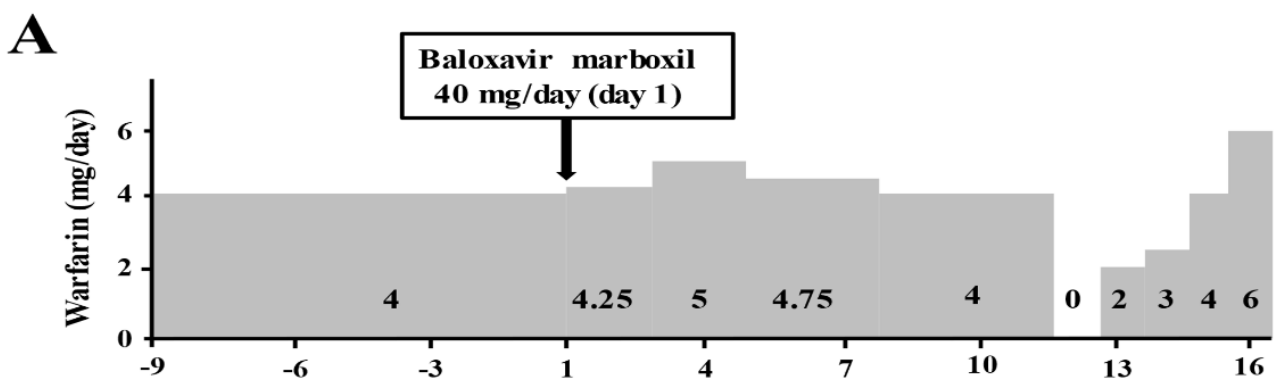

B

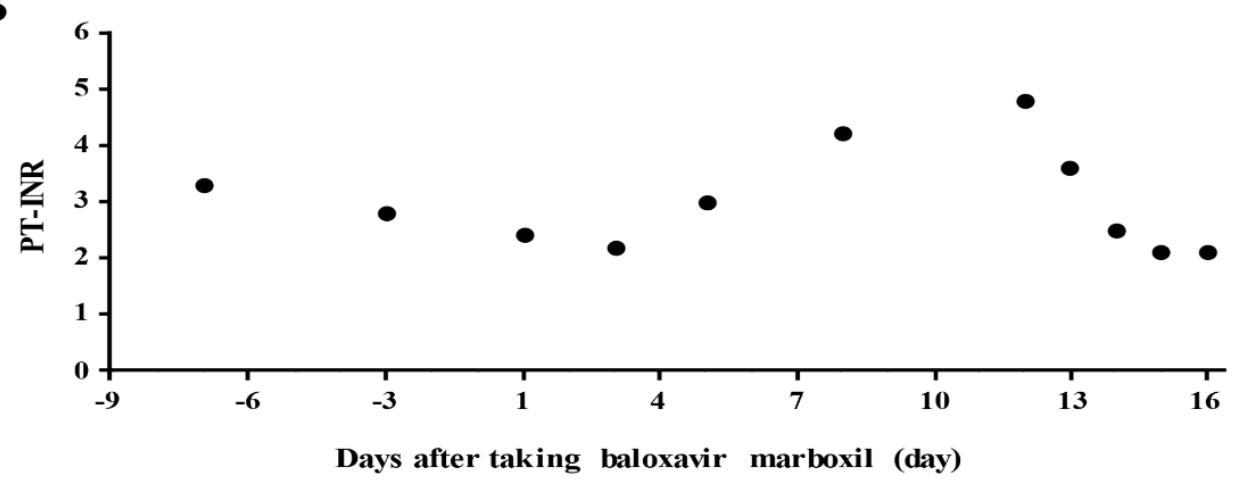

Figure 1. Changes in warfarin dose (A) and prothrombin time-international normalized ratio (PT-INR) (B) before and after taking baloxavir marboxil (BM). A patient on implantable ventricular assist device (iVAD) taking warfarin was diagnosed with influenza and was administered BM. The PT-INR was increased.

He showed good drug compliance and did not take foods or supplements rich in vitamin $\mathrm{K}$ that would affect the effects of warfarin. His other concomitant medications were clopidogrel sulfate, vonoprazan fumarate, eplerenone, carvedilol, enalapril maleate, furosemide, tolvaptan, amiodarone hydrochloride, sodium ferrous citrate, Clostridium butyricum MIYAIRI, magnesium oxide, quetiapine fumarate, pregabalin, olopatadine hydrochloride, and suvorexant. The changes in his laboratory tests (mean \pm standard deviation) before and after taking BM were as follows: PT-INR, $3.2 \pm$ $1.0 ; \mathrm{Hb}, 15.5 \pm 1.1 \mathrm{~g} / \mathrm{dL}$; total bilirubin, $1.2 \pm 0.3$ $\mathrm{mg} / \mathrm{dL}$; gamma-glutamyltransferase, $69.0 \pm 9.9 \mathrm{U} / \mathrm{L}$;
AST, $20.5 \pm 2.1 \mathrm{U} / \mathrm{L} ;$ ALT, $13.5 \pm 2.1 \mathrm{U} / \mathrm{L}$; albumin, $4.1 \pm 0.1 \mathrm{~g} / \mathrm{dL}$; serum creatinine, $0.8 \pm 0.1 \mathrm{mg} / \mathrm{dL}$; and estimated glomerular filtration rate, $78.5 \pm 6.4$ $\mathrm{mL} / \mathrm{min} / 1.73 \mathrm{~m}^{2}$.

\section{DISCUSSION}

To the best of our knowledge, this is the first case report to show that the anti-influenza drug BM increased PT-INR in a patient on iVAD taking warfarin. In this case, based on the history of BM administration and clinical course, the increase in PT-INR could be due to BM. Warfarin interacts with various drugs and foods and inhibits vitamin $\mathrm{K}$ - 
dependent coagulation factors. It is metabolized by cytochrome P450 (CYP450) isozymes; furthermore, it has a high protein-binding rate [6-9]. BM is immediately hydrolyzed to its active form, S-033447 [1], which has a high protein-binding rate $(93 \%-$ 94\%). S- 033447 is mainly metabolized by uridine diphosphate glucuronosyltransferase $1 \mathrm{~A} 3$ to a glucuronide conjugate, and then by CYP3A to sulfoxide, which is excreted in the bile. Its half-life of is estimated to be $96 \mathrm{~h} \mathrm{[1].} \mathrm{In} \mathrm{addition,} \mathrm{BM} \mathrm{and} \mathrm{S-}$ 033447 are substrates for P-glycoprotein (P-gp). BM weakly inhibits P-gp, CY2B6, CYP2C8, and CYP3A, and S-033447 inhibits P-gp and breast cancer receptor protein, but they have no major clinical effect [1]. Therefore, we suspected that the BMinduced increase in PT-INR in our patient involved competition for protein-binding sites as reported for antimicrobials [6-8]. Another possible reason could be that the symptoms of influenza had reduced the intake of diets containing vitamin K. Oseltamivir, an oral anti-influenza drug with neuraminidase inhibitory effect, was initially reported to have no effect on the pharmacokinetics and pharmacodynamics of warfarin [10]. Recently, it has been reported that oseltamivir increased the PT-INR in patients receiving warfarin [11-14]. For reasons that have not been clarified, it is recommended to closely monitor the PT-INR when using oseltamivir in patients taking warfarin [11-14].

Another important point in this case was the early detection of his elevated PT-INR by POCT. It is crucial to control PT-INR by POCT in patients taking warfarin [15], and its usefulness in patients on iVAD has been confirmed [16]. It is used by patients on iVAD in Japan. When the risk of blood clot is high and the target range of PT-INR is high as in this case, the risk of bleeding should be considered. Therefore, interaction with warfarin is a serious problem and frequent PT-INR measurements by POCT are essential.

\section{CONCLUSION}

Strict monitoring of PT-INR is necessary when BM is administered to patients taking warfarin. More caution is needed especially when treating high-risk patients with a high PT-INR target range.

\section{AUTHORS CONTRIBUTION}

All authors meet the ICMJE authorship criteria. KK was the main contributor in the conception and preparation of the manuscript. KK, HS, M Akiyama,
M Akiba, and YS participated in the treatment of the patient. ST revised the manuscript critically. ST and YS collected drug information on the interaction. NM supervised the conception and the manuscript. All authors read and approved the final manuscript.

\section{CONFLICT OF INTEREST}

The authors have no competing interests to declare.

\section{FUNDING}

This work did not receive any funding.

\section{REFERENCES}

1. Heo YA. Baloxavir: first global approval. Drugs 2018;78:693-7. https://doi.org/10.1007/s40265-018-0899-1.

2. Hayden FG, Sugaya N, Hirotsu N, Lee N, De Jong $\mathrm{MD}$, Hurt $\mathrm{AC}$, et al. Baloxavir marboxil for uncomplicated influenza in adults and adolescents. N Engl J Med 2018;379:913-23. https://doi.org/10.1056/NEJMoa1716197.

3. Ison MG, Portsmouth S, Yoshida $\mathrm{Y}$, Shishido T, Mitchener M, Tsuchiya K, et al. Early treatment with baloxavir marboxil in high-risk adolescent and adult outpatients with uncomplicated influenza (CAPSTONE-2): a randomised, placebocontrolled, phase 3 trial. Lancet Infect Dis 2020;3099. https://doi.org/10.1016/s14733099(20)30004-9.

4. Fukushima N, Ono M, Saiki Y, Sawa Y, Nunoda S, Isobe M. Registry report on heart transplantation in Japan (June 2016). Circ J 2017;81:298-303. https://doi.org/10.1253/circj.CJ-16-0976.

5. Eckman PM, John R. Bleeding and thrombosis in patients with continuous-flow ventricular assist devices. Circulation 2012;125:3038-47.

https://doi.org/10.1161/CIRCULATIONA HA.111.040246.

6. Vranckx P, Valgimigli M, Heidbuchel $\mathrm{H}$. The significance of drug-drug and drug-food interactions of oral anticoagulation. Arrhythmia Electrophysiol Rev 2018;7:5561. https://doi.org/10.15420/aer.2017.50.1.

7. Vazquez SR. Drug-drug interactions in an era of multiple anticoagulants: A focus on clinically relevant drug interactions. 
Hematology 2018;2018:339-47. https://doi.org/10.1182/asheducation2018.1.339.

8. Carpenter M, Berry H, Pelletier AL. Clinically relevant drug-drug interactions in primary care. Am Fam Physician 2019;99:558-64.

9. Tan CSS, Lee SWH. Warfarin and food, herbal or dietary supplement interactions: A systematic review. Br J Clin Pharmacol 2020. https://doi.org/10.1111/bcp.14404.

10. Davies BE, Baldó PA, Lennon-Chrimes S, Brewster M. Effect of oseltamivir treatment on anticoagulation: A cross-over study in warfarinized patients. Br J Clin Pharmacol 2010;70:834-43.

https://doi.org/10.1111/j.1365-

2125.2010.03780.x.

11. Lee SH, Kang HR, Jung JW, Kwon JW, Hong KS, Yu KS, et al. Effect of oseltamivir on bleeding risk associated with warfarin therapy: A retrospective review. Clin Drug Investig 2012;32:131-7. https://doi.org/10.2165/11595860000000000-00000.

12. Wagner J, Abdel-Rahman SM. Oseltamivirwarfarin interaction in hypoplastic left heart syndrome: Case report and review. Pediatrics 2015;135:e1333-6. https://doi.org/10.1542/peds.2014-2578.
13. Shah SP, Patel KM, Subedi R, Gambhir HS. Review of potential drug interaction between Oseltamivir and Warfarin and why it is important for emergency medicine physicians. Am J Emerg Med 2017;35:1207.e3-1207.e4. https://doi.org/10.1016/j.ajem.2017.03.040.

14. Smith KR, Bryan WE, Townsend ML, Randolph AE, Vanderman AJ, Woodard CL, et al. Impact of prophylactic oseltamivir on INR in patients on stable warfarin therapy. J Thromb Thrombolysis 2020. https://doi.org/10.1007/s11239-019-020163.

15. Matchar DB, Jacobson A, Dolor R, Edson R, Uyeda L, Phibbs CS, et al. Effect of home testing of international normalized ratio on clinical events. N Engl J Med 2010;363:1608-20. https://doi.org/10.1056/NEJMoa1002617.

16. Bishop MA, Streiff MB, Ensor CR, Tedford RJ, Russell SD, Ross PA. Pharmacistmanaged international normalized ratio patient self-testing is associated with increased time in therapeutic range in patients with left ventricular assist devices at an academic medical center. ASAIO J 2014. https://doi.org/10.1097/MAT.00000000000 00047. 